\title{
Effectiveness of Formal Dysphagia Screening for Stroke Patients
}

\author{
Mi Ran Yoo, M.D. ${ }^{1}$, Yoon Mok Chun, M.D. ${ }^{1}$, Kyoung Hyo Choi, M.D. ${ }^{1}$, \\ Sun Uck Kwon, M.D. ${ }^{2}$, Eun-Jae Lee, M.D. ${ }^{2}$ \\ ${ }^{1}$ Department of Rehabilitation Medicine, Asan Medical Center, University of Ulsan College of Medicine, Seoul, \\ ${ }^{2}$ Department of Neurology, Asan Medical Center, University of Ulsan College of Medicine, Seoul, Korea
}

\begin{abstract}
Objective: Early identification of dysphagia after stroke helps in preventing aspiration pneumonia. However, data are limited regarding the effectiveness of formal dysphagia screening for reducing the risk of aspiration pneumonia. The current study evaluates the effectiveness of formal dysphagia screening in stroke patients, to prevent future episodes of aspiration pneumonia.

Methods: The stroke registry of a tertiary hospital was retrospectively reviewed. We compared clinical variables and the incidence of aspiration pneumonia of patients hospitalized between 2014 and 2015 after formal screening was implemented, and patients hospitalized in 2011 when no established dysphagia screening protocol was in place. Additionally, we identified the incidence of pneumonia according to stroke severity, and evaluated the association with results obtained for incidence of pneumonia and dysphagia screening.

Results: A total of 2,902 patients were identified to have suffered acute stroke (2,018 who underwent formal dysphagia screening; 884 without screening). Patients with formal dysphagia screening developed pneumonia less frequently than patients not administered screening ( $1.3 \%$ with formal screening vs. $3.4 \%$ no formal screening, $\mathrm{P}<0.001)$. Pneumonia was significantly lower in patients with moderate and severe stroke who underwent formal dysphagia screening. Furthermore, failure of the dysphagia screening test, presentation with severe dysarthria, and conditions where dysarthria could not be evaluated, were independent predictors of pneumonia among patients who underwent formal screening.

Conclusion: Our findings demonstrate the association of formal dysphagia screening with reduced risk of poststroke aspiration pneumonia, and indicates the efficacy of the procedure in identifying patients at higher risk of contracting pneumonia. (JKDS 2021;11:43-51)
\end{abstract}

Keywords: Dysphagia, Screening, Stroke, Pneumonia, Aspiration

\section{INTRODUCTION}

Dysphagia after stroke is a common neurological symptom; $35-67 \%$ of acute stroke patients develop dysphagia ${ }^{1,2}$. Aspiration of food or oral secretions and dysfunction of the cough reflex in patients with dysphagia can lead to pneumonia. Stroke-associated pneumonia has been found to correlate with longer
Received: July 20 2020, Revised: July 21 2020,

Accepted: August 282020

Corresponding author: Kyoung Hyo Choi, Department of Rehabilitation Medicine, Asan Medical Center, University of Ulsan College of Medicine, 88 Olympic-ro 43-gil, Songpa-gu, Seoul 05505, Korea Tel: +82-2-3010-3797, Fax: +82-2-3010-6964

E-mail: kyounghyochoi@gmail.com
Copyrights (c) The Korean Dysphagia Society, 2021. 
hospital stays, worse prognosis, and increased healthcare costs of stroke patients ${ }^{1-5}$.

To prevent the above-mentioned problems caused by dysphagia in acute stroke patients, various methods can be utilized to identify dysphagia early. Methods for identifying dysphagia include dysphagia screening, which includes a swallowing test that can be conducted at the bedside or in-office, and more detailed dysphagia assessments, such as videofluoroscopic swallowing study (VFSS) and fiberoptic endoscopic examination of swallowing (FEES). Despite the effectiveness of these and other tests for identifying dysphagia, it is difficult to apply the above methods to all stroke patients due to limitations in manpower and cost, so dysphagia screening is often selectively applied to patients who are more likely to have dysphagia ${ }^{3-6}$.

Although it is not clear whether different methods of swallowing assessments reduce the risk of pneumonia, disability, or death after stroke, the bedside screening test for dysphagia has been shown to effectively reduce pneumonia incidence rates when applied to patients with acute stroke $e^{6-9}$.

Additionally, dysphagia screening before stroke patients begin oral intake has been recommended ${ }^{8}$. In several studies, implementation of dysphagia screening programs has been shown to reduce the incidence of pneumonia ${ }^{10,11}$. Moreover, the use of a formal dysphagia screening protocol in a prospective 15-hospital study was reported to lead to a decrease in the incidence of pneumonia in stroke patients ${ }^{12}$.

Despite the recommendation of stroke management guideline ${ }^{8}$, the evidence level for recommendation is moderate grade, and insufficient evidence has been provided to establish that dysphagia screening protocols effectively improve patient outcomes, such as by reducing pneumonia incidence ${ }^{8,13}$. In a recent study, no association between the use of dysphagia screening and detailed dysphagia assessment and the risk of adverse outcomes including pneumonia $^{14}$.

We wanted to confirm whether the implementation of a formal dysphagia screening program where all acute stroke patients regardless of severity are screened can effectively reduce the incidence of pneumonia in stroke patients in our hospital. We also investigated the association between dysphagia screening result and incidence of pneumonia. The purpose of this study was to evaluate the effectiveness of formal dysphagia screening in patients with stroke for the prevention of aspiration pneumonia.

\section{MATERIALS AND METHODS}

\section{Study design}

We retrospectively identified stroke patients who were hospitalized in the neurology department of a single tertiary hospital. All stroke (ischemic stroke, hemorrhagic stroke) diagnoses were confirmed by admitting neurologists. Patients who died during the hospitalization period were excluded.

Dysphagia screening was defined as the use of a simple non-invasive bedside swallow screening test. Until 2012, dysphagia screenings were performed by admitting neurologists without an established dysphagia screening protocol. Also, the tests have been only applied for stroke patients with higher stroke severity.

A standardized dysphagia screening protocol was implemented in our institution in 2014. Since then, all stroke patients underwent documented dysphagia screening within 48 hours of hospital admission by a trained speech-language pathologist (SLP). The patients were checked for mental status, dysarthria, facial palsy, and tongue impairment or dysphonia. And a dry swallow test was first performed and then a wet swallow test where the patient was instructed to drink a small volume of water was performed. The formal dysphagia screening tests have been routinely performed in all stroke patients regardless of stroke severity since 2014 .

We compared screening results and outcomes of stroke patients who underwent formal dysphagia screening (classified as the 'formal screening group' of patients who were hospitalized between 2014 and 2015) and those who did not receive formal dysphagia 
screening (classified as the 'no formal screening group' of patients who were hospitalized in 2011).

Stroke patients who underwent formal dysphagia screening were further categorized into those who passed or failed the test so that outcomes could be compared. If the patient failed to swallow or swallowed with choking, wet hoarseness or changes in breathing in a dry swallow test or wet swallow test, each test was evaluated as failed test. If the patient failed either the dry swallow test or the wet swallow test, the patient was categorized into the group who failed the test.

This study was approved by the center's institutional review board (S2017-1210-0003).

\section{Data and outcome variables}

Demographic variables included age, sex, stroke type, stroke severity score by the National Institutes of Health Stroke Scale (NIHSS), stroke risk factors (heart disease, diabetes mellitus, hypertension, dyslipidemia), level of consciousness (alert, not alert), presentation with dysarthria and modified Rankin Scale on admission.

Initial NIHSS scores were categorized into three groups: mild (NIHSS 0-4), moderate (NIHSS 5-15), severe (NIHSS 16-42).

The outcome of interest was the incidence of radiographically confirmed aspiration pneumonia within 30 days of hospitalization.

\section{Diagnosis of aspiration pneumonia}

Aspiration pneumonia was confirmed when all of the following conditions were met: (1) the patient developed new-onset fever; (2) at the time of fever occurrence, pneumonia or aspiration pneumonia was recorded as the cause of fever in the medical record, including the progress notes and/or consultation notes; (3) piperacillin/tazobactam was administered at the time of fever occurrence; (4) abnormal lab findings such as leukopenia, leukocytosis, or elevated C-reactive protein were identified; and (5) definitive abnormal findings on chest radiographs were identified within 30 days of hospitalization ${ }^{15,16}$.
Patients were excluded if they: (1) were transferred from another hospital after receiving antibiotics, (2) had another potential fever origin such as urinary tract infection or thrombophlebitis, (3) or were diagnosed with community-acquired pneumonia rather than hospital-acquired pneumonia.

\section{Statistical analysis}

Categorical variables are presented as percentages and continuous or discrete variables are presented as means \pm standard deviations. To compare the characteristics and the outcome of interest, the Student's t-test was used for continuous variables and Fisher's exact test and the chi-squared test were used for categorical variables.

The association between clinical variables and incidence of aspiration pneumonia was assessed by univariate logistic regression analysis. For clinical variables with $P$ values $<0.1$ were included in multivariable models. All potentially significant variables were included in multivariate logistic regression analysis. Multiple logistic regression analysis with stepwise fashioned variable selection was used to identify variables that were independent predictors of incidence of aspiration pneumonia.

The primary analysis was used to evaluate associations between the use of formal dysphagia screening on the outcome of aspiration pneumonia. We also calculated the proportion of patients who failed the screening test. For the secondary analysis, we used multiple logistic regression to identify the effect of failing screening on the odds of patients developing pneumonia, with adjustment of potential confounders.

All statistical analyses were performed using SPSS version 18.0 (IBM Inc., Armonk, NY, USA). Differences were considered to be statistically significant when $\mathrm{P}<0.05$.

\section{RESULTS}

Between January 1st, 2011, and December 31st, 2011, 901 patients who were admitted to the neurology department for stroke without undergoing for- 
mal dysphagia screening were identified; the medical charts of 12 patients were irretrievable and 5 patients were excluded ( 1 was admitted for another reason and 4 died during the hospitalization period), leaving 884 patients to be included in the no formal screening group. Between January 1st, 2014, and December 31st, 2015, 2,054 stroke patients were admitted and underwent formal dysphagia screening; the medical charts of 27 patients were irretrievable and 9 patients were excluded because they were admitted for another reason, leaving 2,018 patients in the formal screening group.(Fig. 1)

Table 1 shows the comparison of clinical variables between patients who did or did not undergo formal dysphagia screening. There were no significant difference in the distribution of sex, age, hypertension, diabetes mellitus status, cardiac disease, dyslipidemia, level of consciousness, presentation with dysarthria, mean NIHSS stroke severity score, and modified Rankin Scale at admission between the two groups. There was also no significant difference in the distribution of NIHSS scores between the groups of patients who either did or did not undergo formal dysphagia screening. There were significantly more ischemic stroke patients in the formal dysphagia screening group than the no formal screening group (ischemic stroke with no formal screening, 90.4\% (799/884) vs. formal screening, 93.6\% (1,889/2,018); $\mathrm{P}=0.004)$. Meanwhile, when comparing the two groups, the proprotions of patients receiving non-oral diet was similar in both groups, but the proportion of patients receiving a modified diet was higher in the formal dysphagia screening group (non-oral diet with no formal screening, 17.6\% (156/884) vs. formal screening, $16.2 \%$ (326/2018), modified diet with no formal screening, $28.9 \%(255 / 884)$ vs. formal screening, $38.5 \%$ (777/2,018); $\mathrm{P}=0.01$ ).

The proportion of patients diagnosed with aspiration pneumonia was significantly lower in the formal dysphagia screening group than in the no formal screening group $(1.3 \%(26 / 2,018)$ vs. $3.4 \%(30 / 884) ; \mathrm{P}<$ 0.001).

The subgroup analysis of patients diagnosed with aspiration pneumonia according to NIHSS group is shown in Table 2. The proportion of patients diagnosed with aspiration pneumonia was significantly

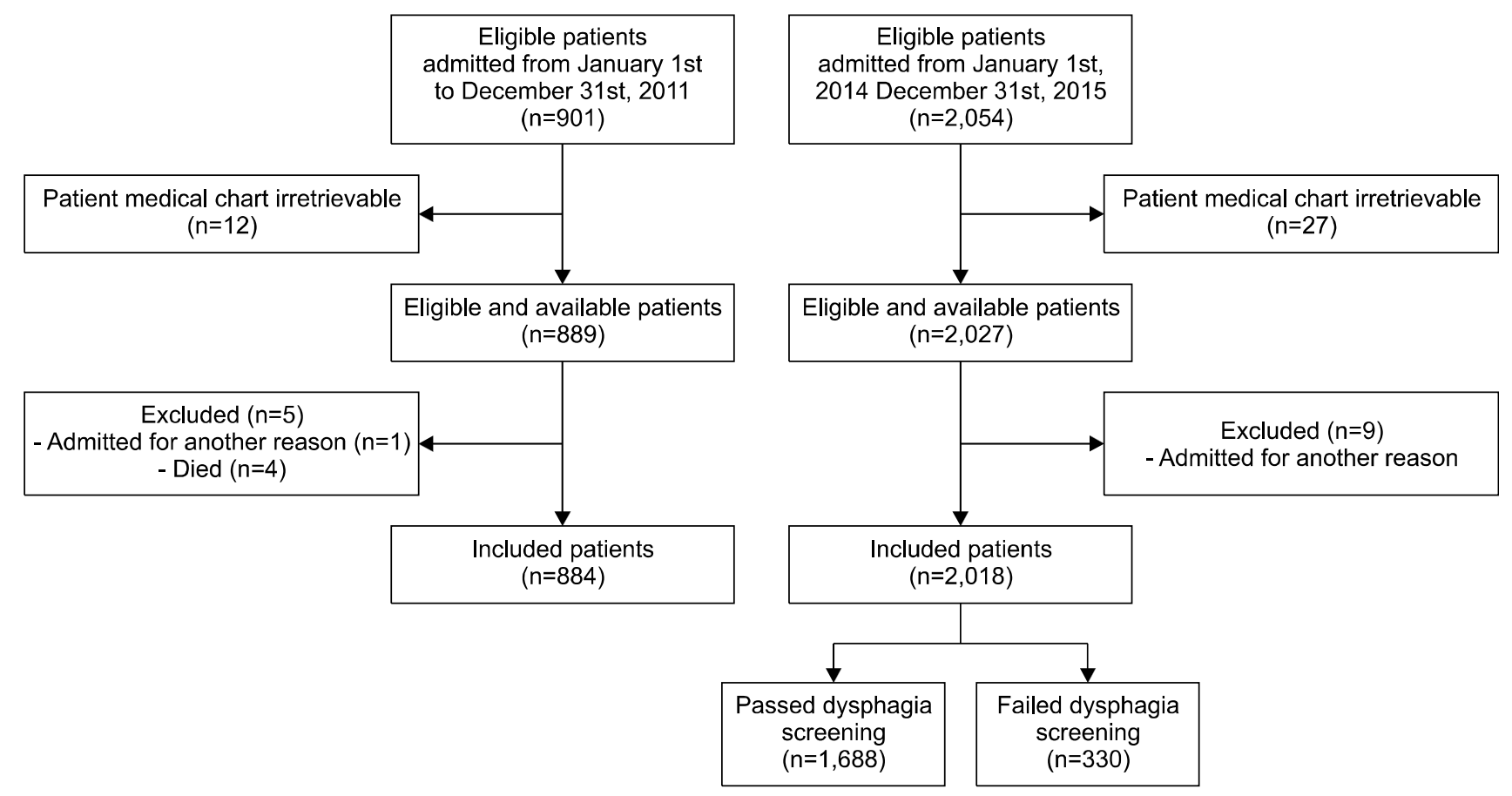

Fig. 1. Flow diagram of patients did or did not undergo formal dysphagia screening. 
Table 1. Patient characteristics and incidence of pneumonia of patients who did or did not undergo formal dysphagia screening.

\begin{tabular}{|c|c|c|c|}
\hline Variable & $\begin{array}{c}\text { No formal } \\
\text { screening group } \\
\quad(n=884)\end{array}$ & $\begin{array}{l}\text { Formal } \\
\text { screening group } \\
(\mathrm{n}=2018)\end{array}$ & $\mathrm{P}$ value \\
\hline \multicolumn{4}{|l|}{ Sex } \\
\hline Female/Male & $340 / 544$ & $770 / 1248$ & 0.88 \\
\hline Age & $64.7 \pm 12.9$ & $65.2 \pm 13.6$ & 0.24 \\
\hline Stroke type & & & $0.004^{*}$ \\
\hline Ischemic & 799 (90.4\%) & 1889 (93.6\%) & \\
\hline Hemorrhagic & $85(9.6 \%)$ & $129(6.4 \%)$ & \\
\hline Pneumonia & $30(3.4 \%)$ & $26(1.3 \%)$ & $<0.001^{*}$ \\
\hline Hypertension & $567(64.1 \%)$ & $1246(61.8 \%)$ & 0.23 \\
\hline Diabetes mellitus & $255(28.8 \%)$ & 577 (28.6\%) & 0.89 \\
\hline Cardiac disease & $273(30.9 \%)$ & $588(28.2 \%)$ & 0.35 \\
\hline Dyslipidemia & $221(25.0 \%)$ & $497(24.7 \%)$ & 0.84 \\
\hline Mental status & & & 0.74 \\
\hline Alert & 817 (92.4\%) & $1872(92.8 \%)$ & \\
\hline Non alert & $67(7.6 \%)$ & $146(7.2 \%)$ & \\
\hline Dysarthria & & & 0.73 \\
\hline None or mild & $607(68.7 \%)$ & $1404(69.6 \%)$ & \\
\hline Severe & $197(22.3 \%)$ & $449(22.2 \%)$ & \\
\hline Undeterminable & $80(9.0 \%)$ & $165(8.2 \%)$ & \\
\hline Mean NIHSS score & $5.08 \pm 5.4$ & $5.24 \pm 5.1$ & 0.20 \\
\hline NIHSS group & & & 0.66 \\
\hline Mild & $520(58.8 \%)$ & $1159(57.4 \%)$ & \\
\hline Moderate & 304 (34.4\%) & $730(36.1 \%)$ & \\
\hline Severe & $60(6.8 \%)$ & $130(6.4 \%)$ & \\
\hline mRS at admission & & & 0.51 \\
\hline $0-2$ & $472(53.4 \%)$ & $1104(54.7 \%)$ & \\
\hline $3-5$ & $412(46.6 \%)$ & $914(45.3 \%)$ & \\
\hline Diet recommendation & & & $0.01^{*}$ \\
\hline Non-oral & $156(17.6 \%)$ & $326(16.2 \%)$ & \\
\hline Modified diet & 255 (28.9\%) & 777 (38.5\%) & \\
\hline Regular diet & $473(53.5 \%)$ & 915 (45.3\%) & \\
\hline
\end{tabular}

*** $\mathrm{P}<0.05$, statistically significant by Chi-squared test.

NIHSS: National Institutes of Health Stroke Scale, mRS: modified Rankin Scale.

lower in the formal dysphagia screening group when compared to the no formal screening group among moderate and severe stroke patients (moderate, no formal screening $5.4 \%$ vs. formal screening $1.5 \%$, $\mathrm{P}=0.005$; severe, no formal screening $4.2 \%$ vs. formal screening 2.6\%; $\mathrm{P}=0.02$ ); there was no significant difference in the proportions of mild stroke patients diagnosed with aspiration pneumonia between the two groups (no formal screening $0.3 \%$ vs. formal screening $0.2 \%$; $\mathrm{P}=0.11$ ).

Table 3 shows the univariate and multivariate logistic regression analyses of clinical variables associated with aspiration pneumonia incidence. In the
Table 2. Subgroup analysis of aspiration pneumonia incidence according to NIHSS group.

\begin{tabular}{|c|c|c|c|}
\hline NIHSS group & $\begin{array}{c}\text { No formal } \\
\text { screening group } \\
(\mathrm{n}=884)\end{array}$ & $\begin{array}{c}\text { Formal } \\
\text { screening group } \\
(\mathrm{n}=2018)\end{array}$ & $P$ value \\
\hline Mild ( $n=1679)$ & $5(0.3 \%)$ & $10(0.2 \%)$ & 0.11 \\
\hline Moderate $(n=1033)$ & $17(5.4 \%)$ & $16(1.5 \%)$ & $0.005^{*}$ \\
\hline Severe $(n=190)$ & $8(4.2 \%)$ & $3(2.6 \%)$ & $0.02^{\text {*** }}$ \\
\hline
\end{tabular}

univariate logistic regression analysis, increasing age, level of consciousness (not alert), increasing stroke severity indicated by NIHSS score, modified Rankin Scale (mRS) at admission (3-5), presentation with dysarthria (severe or undeterminable) and not receiving formal dysphagia screening were significantly associated with the development of aspiration pneumonia. Not receiving formal dysphagia screening, lower level of function according to mRS scores, severe dysarthria, and conditions where dysarthria cannot be evaluated were independent predictors of pneumonia in the multivariate regression analysis. The adjusted odds ratio of developing pneumonia in patients who underwent dysphagia screening was 0.36 (95\% CI, 0.20 to 0.62 ).

Table 4 shows the baseline characteristics of patients who either passed or failed the formal dysphagia screening test. Of the 2,018 patients who underwent formal dysphagia screening, 330 (16.4\%) patients failed. Patients who failed screening had lower levels of consciousness, higher stroke severity, more frequent severe dysarthria, lower levels of function according to mRS scores, and were more often placed on a non-oral diet regime. The proportion of patients diagnosed with aspiration pneumonia was significantly higher in the patients who failed the dysphagia screening test than in the patients who passed the screening test $(5.5 \%(4 / 330)$ vs. $0.4 \%(21 / 1688) ; \mathrm{P}<$ 0.001).

The univariate and multivariate logistic regression analyses for incidence of aspiration pneumonia of patients who underwent formal dysphagia screening 
Table 3. Univariate and multivariate logistic regression analyses of clinical variables for association with aspiration pneumonia incidence.

\begin{tabular}{|c|c|c|c|c|c|c|}
\hline & \multicolumn{3}{|c|}{ Univariate analysis } & \multicolumn{3}{|c|}{ Multivariate analysis } \\
\hline & OR & $95 \%$ CI & $P$ value & OR & $95 \%$ CI & $P$ value \\
\hline Formal dysphagia screening & 0.37 & $0.22-0.63$ & $<0.000^{*}$ & 0.36 & $0.20-0.62$ & $<0.000^{*}$ \\
\hline Age & 1.04 & $1.01-1.06$ & $<0.002^{*}$ & 1.03 & $1.01-1.06$ & 0.51 \\
\hline Sex (Male) & 0.89 & $0.51-1.54$ & 0.67 & & & \\
\hline \multicolumn{7}{|l|}{ Stroke type } \\
\hline Ischemic & Reference & - & - & - & - & - \\
\hline Hemorrhagic & 2.43 & $1.17-5.02$ & 0.10 & 1.99 & $0.93-4.27$ & 0.08 \\
\hline \multicolumn{7}{|l|}{ Mental status } \\
\hline Alert & Reference & - & - & - & - & - \\
\hline Not alert & 5.52 & $3.03-10.1$ & $<0.000^{*}$ & 1.18 & $0.58-2.41$ & 0.65 \\
\hline Stroke severity (NIHSS) & 1.16 & $1.12-1.20$ & $<0.000^{*}$ & 1.12 & $0.95-1.09$ & 0.64 \\
\hline \multicolumn{7}{|l|}{ mRS at admission } \\
\hline $0-2$ & Reference & - & - & - & - & - \\
\hline $3-5$ & 8.41 & $3.80-18.70$ & $<0.000^{*}$ & 2.77 & $1.12-6.83$ & $0.027^{*}$ \\
\hline \multicolumn{7}{|l|}{ Dysarthria } \\
\hline None or mild & Reference & - & - & - & - & - \\
\hline Severe & 5.67 & $2.86-11.3$ & $<0.000^{*}$ & 2.40 & $1.09-5.29$ & $0.030^{*}$ \\
\hline Undeterminable & 12.9 & $6.30-26.5$ & $<0.000^{*}$ & 2.85 & $0.99-8.19$ & $0.049 *$ \\
\hline
\end{tabular}

$* \mathrm{P}<0.05$, statistically significant.

NIHSS: National Institutes of Health Stroke Scale, mRS: modified Rankin Scale, OR: odds ratio, CI: confidence interval.

are shown in Table 5. In the multivariate analysis, compared to those who passed screening, patients who failed screening had a significantly higher risk of pneumonia (5.5\% vs. $0.4 \%$; aOR, 5.53 ; $95 \% \mathrm{CI}, 1.87-$ 16.4). Meanwhile, presentation with severe dysarthria and conditions where dysarthria could not be evaluated were found to be independent predictors of pneumonia in the multivariate regression analysis (22.2\%; aOR, 4.48; 95\% CI, 1.25-16.04; 8.2\%; aOR, 1.50; 95\% CI, 1.49-26.70, respectively).

\section{DISCUSSION}

We found that formal dysphagia screening is associated with lower risk of pneumonia in acute stroke patients when compared to acute stroke patients who did not undergo formal screening. Furthermore, among patients who underwent formal dysphagia screening, failing a dysphagia screening test, presentation with severe dysarthria, and conditions where dysarthria cannot be evaluated were independent predictors of aspiration pneumonia.

Although many efforts have been made to implement early screening for dysphagia in stroke patients, the adherence rates for dysphagia screening protocols remains low, and some acute stroke units still have no formal screening protocol ${ }^{12}$. Meanwhile, there is insufficient evidence that the formal dysphagia screening protocol effectively improves patient outcomes.

Since 2014, our institution has followed a formal dysphagia screening protocol and it was performed in stroke patients of all severity levels including mild stroke at the time of admission to the neurology department. Before 2012, dysphagia screening was only used for patients with higher stroke severity levels. Notably, our study identified significantly fewer aspiration pneumonia cases among patients after formal dysphagia screening was implemented. We found that implementing the formal dysphagia screening reduced the incidence of aspiration pneumonia, even after adjusting for stroke severity.

These findings are similar to those of a previous study that also found that implementation of a formal dysphagia screening protocol was associated with reduced risk of pneumonia ${ }^{12}$. Other institutions have also implemented dysphagia screening programs with similar results. For example, Yeh et al. reported that 
Table 4. Characteristics of patients who passed or failed the formal dysphagia screening test.

\begin{tabular}{|c|c|c|c|}
\hline \multirow{2}{*}{ Variable } & \multicolumn{2}{|c|}{ Dysphagia screening result } & \multirow{2}{*}{$P$ value } \\
\hline & Pass & Fail & \\
\hline N (\%) & $1,688(83.6 \%)$ & $330(16.4 \%)$ & \\
\hline \multicolumn{4}{|l|}{ Sex } \\
\hline Female/Male & $644 / 1,044$ & $126 / 204$ & 0.11 \\
\hline Age & $64.9 \pm 13.5$ & $67.8 \pm 13.5$ & 0.73 \\
\hline Stroke type & & & 0.23 \\
\hline Ischemic & $1,580(93.9 \%)$ & 309 (92.1\%) & \\
\hline Hemorrhagic & $108(6.1 \%)$ & $21(7.9 \%)$ & \\
\hline Pneumonia & $21(0.4 \%)$ & $4(5.5 \%)$ & $<0.001^{* * * *}$ \\
\hline Hypertension & $1,042(61.8 \%)$ & $204(61.5 \%)$ & 0.92 \\
\hline Diabetes mellitus & $483(29.2 \%)$ & $94(25.5 \%)$ & 0.17 \\
\hline Cardiac disease & $492(27.9 \%)$ & $96(29.5 \%)$ & 0.60 \\
\hline Dyslipidemia & $416(25.0 \%)$ & $81(22.7 \%)$ & 0.38 \\
\hline \multicolumn{4}{|l|}{ Mental status } \\
\hline Not alert & $29(1.7 \%)$ & $117(35.5 \%)$ & $<0.001^{*}$ \\
\hline Mean NIHSS score & $3.75 \pm 3.39$ & $12.86 \pm 5.81$ & $<0.001^{* * *}$ \\
\hline NIHSS group & & & $<0.001^{*}$ \\
\hline Mild & $1,127(66.8 \%)$ & $32(9.7 \%)$ & \\
\hline Moderate & $546(32.3 \%)$ & $183(55.5 \%)$ & \\
\hline Severe & $15(0.9 \%)$ & $115(34.8 \%)$ & \\
\hline Dysarthria & & & $<0.001^{*}$ \\
\hline Severe & $314(18.6 \%)$ & 135 (40.9\%) & \\
\hline Undeterminable & $29(1.7 \%)$ & $136(41.2 \%)$ & \\
\hline $\mathrm{mRS}$ at admission & & & $<0.001^{*}$ \\
\hline $3-5$ & $615(36.4 \%)$ & 299 (90.6\%) & \\
\hline Diet recommendation & & & $<0.001^{*}$ \\
\hline Non-oral & $2(0.1 \%)$ & $326(98.8 \%)$ & \\
\hline Modified diet & $773(45.8 \%)$ & $4(1.2 \%)$ & \\
\hline
\end{tabular}

$* \mathrm{P}<0.05$, statistically significant by Chi-squared test, ${ }^{* *} \mathrm{P}<0.05$, statistically significant by Independent $t$-test, ${ }^{* * * * *} \mathrm{P}<0.05$, statistically significant by Fisher's exact test.

NIHSS: National Institutes of Health Stroke Scale.

dysphagia screening was associated with a decrease in stroke-associated pneumonia in all stroke patients after adjusting for factors such as stroke severity ${ }^{17}$. Judith et al. also found that the pneumonia rate was lower in patients who were screened with the formal dysphagia screening process and even after adjusting for stroke severity, and formal dysphagia screening was associated with fewer patients developing pneumonia $^{12}$.

Although patients with mild stroke are not completely without risk for hospital-acquired pneumonia, they are often not considered for dysphagia screening because of their low risk of developing pneumonia. Joundi et al. reported that mild stroke patients were half as likely to be screened as moderate stroke patients. However, patients with mild severity of stroke had moderate rates of dysphagia screening failure and substantial risk of complications after failing the screening test ${ }^{18}$.

The subgroup analysis according to stroke severity score found that aspiration pneumonia was less common among patients with moderate and severe stroke severity after the formal dysphagia screening program was implemented. Although the difference was not significant, aspiration pneumonia was also somewhat lower among mild stroke patients who underwent formal dysphagia screening when compared to patients who did not undergo formal screening.

Several studies have reported that failing the dysphagia screening test is a powerful predictor of poor outcomes such as pneumonia ${ }^{14,18}$, more severe disability $^{14,18}$, and higher likelihood of the patient being discharged to a long-term care facility, even in patients with mild strokes, who are less likely to be screened $^{18}$. The current study also showed that failing the dysphagia screening test is a strong predictor of aspiration pneumonia occurrence, with an adjusted odds ratio of 5.53. Similar odds ratios have been reported in other studies as $4.71^{18}$ and $3.00^{14}$. Furthermore, not only failing a dysphagia screening test, presentation with severe dysarthria and conditions where dysarthria cannot be evaluated were also significant factor which were associated with the incidence of aspiration pneumonia of stroke patients who had underwent the formal dysphagia screening program. These findings were consistent with suggestions from several other studies ${ }^{19,20}$. Therefore, we suggest that formal dysphagia screening can be effective to identify patients at higher risk such as failing the screening or presence of dysarthria for developing poor outcome.

A systematic review of the effects of dysphagia screening strategies on stroke patients found insufficient data supporting the effectiveness of implementing a dysphagia screening protocol, however, only three studies were included and because of the limited research available and no definite conclusions 
Table 5. Univariate and multivariate logistic regression analyses for incidence of aspiration pneumonia of patients who underwent routine dysphagia screening.

\begin{tabular}{|c|c|c|c|c|c|c|}
\hline & \multicolumn{3}{|c|}{ Univariate analysis } & \multicolumn{3}{|c|}{ Multivariate analysis } \\
\hline & OR & $95 \% \mathrm{CI}$ & $\mathrm{P}$ value & OR & $95 \% \mathrm{CI}$ & $P$ value \\
\hline Sex (Male) & 0.89 & $0.51-1.54$ & 0.67 & & & \\
\hline \multicolumn{7}{|l|}{ Mental status } \\
\hline Alert & Reference & - & - & - & - & - \\
\hline Not alert & 6.33 & $2.68-14.92$ & $<0.000^{*}$ & 1.45 & $0.54-3.95$ & 0.46 \\
\hline Stroke severity (NIHSS) & 1.16 & $1.12-1.20$ & $<0.000^{*}$ & 0.97 & $0.88-1.08$ & 0.61 \\
\hline \multicolumn{7}{|l|}{ mRS at admission } \\
\hline $0-2$ & Reference & - & - & - & - & - \\
\hline $3-5$ & 8.41 & $3.80-18.70$ & $<0.000^{*}$ & 1.25 & $0.30-5.27$ & 0.76 \\
\hline \multicolumn{7}{|l|}{ Dysarthria } \\
\hline None or mild & Reference & - & - & - & - & - \\
\hline Severe & 8.79 & $2.86-27.3$ & $<0.000^{*}$ & 4.48 & $1.25-16.04$ & $0.021^{*}$ \\
\hline Undeterminable & 12.90 & $6.30-36.5$ & $<0.000^{*}$ & 1.50 & $1.49-26.70$ & $0.012^{*}$ \\
\hline \multicolumn{7}{|c|}{ Results of dysphagia screen } \\
\hline Pass & Reference & - & - & - & - & - \\
\hline Fail & 13.85 & $5.74-33.45$ & $0.000^{*}$ & 5.53 & $1.87-16.4$ & $0.002 *$ \\
\hline
\end{tabular}

${ }^{*} \mathrm{P}<0.05$, statistically significant.

NIHSS: National Institutes of Health Stroke Scale, mRS: modified Rankin Scale, OR: odds ratio, CI: confidence interval.

could be drawn ${ }^{8}$. Furthermore, there is no standard dysphagia screening protocol and no consensus has been reached regarding which protocols are best ${ }^{21}$; Considering this, it is meaningful that our study used a cost-effective, relatively simple method of clinical examination at the bedside and found that the implementation of this dysphagia screening protocol using a relatively simple method significantly reduced the incidence of pneumonia.

This study had several limitations. First, the sample size between the two groups was quite different which may affect statistical power. However, the Chi-square test, which is robust with respect to the size and distribution of the data, was used to compare the incidence of pneumonia between the two groups. Second, sensitivity, specificity, and accuracy analyses of our formal dysphagia screening program were not performed. However, we wanted to focus on evaluating outcomes after implementing a formal dysphagia screening program, and since there are still many stroke units worldwide that do not yet use formal screening protocol, we believe our study can support the existing stroke guideline recommendation. Third, the dysphagia screening was performed using clinical examinations and water swallowing test that are less sensitive than video fluoroscopic swallowing study and fiberoptic endoscopic examination of swallowing. Finally, due to the retrospective nature of this study, we could not verify pneumonia outcomes beyond the information available in the medical records.

\section{CONCLUSION}

The results of our study suggest that formal dysphagia screening is associated with reduced risk of post-stroke aspiration pneumonia. Among the patients who underwent formal dysphagia screening, failing the dysphagia screening test, presentation with severe dysarthria, and conditions where dysarthria could not be evaluated were associated with increased risk of aspiration pneumonia. Our findings support the implementation of formal dysphagia screening for all stroke patients. Further large-scale prospective studies to confirm the effectiveness of the implementation of formal dysphagia screening for acute stroke patients to prevent aspiration pneumonia are necessary. 


\section{CONFLICT OF INTEREST}

The authors declare that they have no conflict of interest.

\section{ETHICS}

This study was approved by the center's institutional review board (S2017-1210-0003).

\section{ACKNOWLEDGEMENTS}

None.

\section{FUNDING}

None.

\section{REFERENCES}

1. Lakshminarayan K, Tsai AW, Tong X, Vazquez G, Peacock JM, George MG, et al. Utility of dysphagia screening results in predicting poststroke pneumonia. Stroke. 2010;41: 2849-54

2. Martino R, Foley N, Bhogal S, Diamant N, Speechley M, Teasell R. Dysphagia after stroke: incidence, diagnosis, and pulmonary complications. Stroke. 2005;36:2756-63

3. Ramsey DJ, Smithard DG, Kalra L. Early assessments of dysphagia and aspiration risk in acute stroke patients. Stroke. 2003;34:1252-7

4. Al-Khaled M, Matthis C, Binder A, Mudter J, Schattschneider $\mathrm{J}$, Pulkowski U, et al. Dysphagia in patients with acute ischemic stroke: early dysphagia screening may reduce stroke-related pneumonia and improve stroke outcomes. Cerebrovasc Dis. 2016;42:81-9

5. Smith CJ, Kishore AK, Vail A, Chamorro A, Garau J, Hopkins SJ, et al. Diagnosis of stroke-associated pneumonia: recommendations from the pneumonia in stroke consensus group. Stroke. 2015;46:2335-40

6. Trapl M, Enderle P, Nowotny M, Teuschl Y, Matz K, Dachenhausen A, et al. Dysphagia bedside screening for acute-stroke patients: the Gugging Swallowing Screen. Stroke. 2007;38:2948-52

7. Boaden E, Doran D, Burnell J, Clegg A, Dey P, Hurley M, et al. Screening for aspiration risk associated with dysphagia in acute stroke. Cochrane Database Syst Rev. 2017:CD012679

8. Smith EE, Kent DM, Bulsara KR, Leung LY, Lichtman JH, Reeves MJ, et al. Effect of dysphagia screening strategies on clinical outcomes after stroke: a systematic review for the 2018 guidelines for the early management of patients with acute ischemic stroke. Stroke. 2018;49:e1238

9. Titsworth WL, Abram J, Fullerton A, Hester J, Guin P, Waters MF, et al. Prospective quality initiative to maximize dysphagia screening reduces hospital-acquired pneumonia prevalence in patients with stroke. Stroke. 2013; 44:3154-60

10. Odderson IR, Keaton JC, McKenna BS. Swallow management in patients on an acute stroke pathway: quality is cost effective. Arch Phys Med Rehabil. 1995;76:1130-3

11. Doggett DL, Tappe KA, Mitchell MD, Chapell R, Coates V, Turkelson CM. Prevention of pneumonia in elderly stroke patients by systematic diagnosis and treatment of dysphagia: an evidence-based comprehensive analysis of the literature. Dysphagia. 2001;16:279-95

12. Hinchey JA, Shephard T, Furie K, Smith D, Wang D, Tonn S. Formal dysphagia screening protocols prevent pneumonia. Stroke. 2005;36:1972-6

13. Powers WJ, Rabinstein AA, Ackerson T, Adeoye OM, Bambakidis NC, Becker K, et al. Guidelines for the early management of patients with acute ischemic stroke: 2019 update to the 2018 guidelines for the early management of acute ischemic stroke: a guideline for healthcare professionals from the American Heart Association/American Stroke Association. Stroke. 2019;5:e344-418

14. Ouyang M, Boaden E, Arima H, Lavados PM, Billot L, Hackett ML, et al. Dysphagia screening and risks of pneumonia and adverse outcomes after acute stroke: an international multicenter study. Int J Stroke. 2020;15:20615

15. Komiya K, Ishii H, Kadota J. Healthcare-associated pneumonia and aspiration pneumonia. Aging Dis. 2015;6:2737

16. Ottosen J, Evans H. Pneumonia: challenges in the definition, diagnosis, and management of disease. Surg Clin North Am. 2014;94:1305-17

17. Yeh SJ, Huang KY, Wang TG, Chen YC, Chen CH, Tang SC, et al. Dysphagia screening decreases pneumonia in acute stroke patients admitted to the stroke intensive care unit. J Neurol Sci. 2011;306:38-41

18. Joundi RA, Martino R, Saposnik G, Giannakeas V, Fang J, Kapral MK. Predictors and outcomes of dysphagia screening after acute ischemic stroke. Stroke. 2017;48:900-6

19. Bahia MM, Mourão LF, Chun RYS. Dysarthria as a predictor of dysphagia following stroke. NeuroRehabilitation. 2016;38:155-62

20. Wang BJ, Carter FL, Altman KW. Relationship between dysarthria and oral-oropharyngeal dysphagia: the current evidence. Ear Nose Throat J. 2018;97:e1-9

21. Luker JA, Wall K, Bernhardt J, Edwards I, Grimmer-Somers $\mathrm{K}$. Measuring the quality of dysphagia management practices following stroke: a systematic review. Int J Stroke. 2010;5:466-76 